\title{
Research on Maskless Lithography System Based on Digital Oblique Scanning Strategy
}

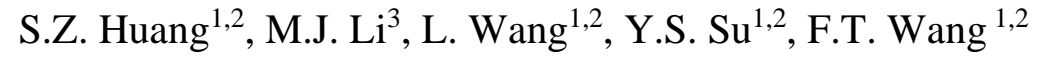 \\ ${ }^{1}$ Key Laboratory of Advanced Perception and Intelligent Control of High-end Equipment, Ministry of \\ Education, Wuhu 241000, China \\ ${ }^{2}$ School of Mechanical and Automotive Engineering, Anhui Polytechnic University, Wuhu 241000, China \\ ${ }^{3}$ Department of Precision Machinery and Precision Instrumentation, University of Science and Technology \\ of China, Hefei 230026, China \\ E-mail:Imn@ustc.edu.cn
}

\begin{abstract}
In this paper, based on the scanning lithography imaging principle of digital micromirror devices (DMD), an oblique scanning lithography strategy was proposed, and successfully used in the maskless lithography system. During the design and construction of the system, ZEMAX optical lighting system design, non-sequential simulation optimization analysis, pulse width modulation and other methods were adopted comprehensively which can effectively improve the lithography quality. The experimental results showed that this lithography system can successfully fabricate large-area linear grating pattern with a resolution of $0.8 \mu \mathrm{m}$, which can effectively make up for the quantization error and other defects existing in the current maskless lithography system, and will have a broad application prospect in fabricating large-area, high-precision micro-nano structures.
\end{abstract}

DOI: $10.2961 /$ jlmn.2020.01.2008

Keywords: digital micromirror devices (DMD), oblique scanning, optical design, lithography system, grating pattern

\section{Introduction}

Compared with traditional laser direct writing, electron beam lithography and other photolithography technologies [1-3], digital micromirror device (DMD)-based maskless lithography [4-6] is due to its advantages of high processing efficiency, low cost, simple process and good processing flexibility, which has been widely concerned by scholars all over the world and is considered as a new digital lithography technology following the single-point laser direct writing technology. At present, it has been widely applied in the micro-optics devices [7], microfluidic biological chips [8], printed circuit boards [9], organic light-emitting diodes (OLED) display [10] and other fields.

In 1999, A. Birtsch research group [11] used DMD as mask generator to replace the liquid crystal display device (LCD), which greatly improved the low-resolution problem of the original system, and the processing resolution could reach about $5 \mathrm{um}$. To further improve the photolithography resolution, Wataru Iwasaki research group [12] combined DMD and high magnification objective lens to build a lithography system stage, which was capable of obtaining submicron resolution and obtaining high-quality lithography graphics with a line width of $500 \mathrm{~nm}$. At present, in order to meet the needs of high-precision machining of large-area microstructures such as printed circuit board (PCB) and large-scale grating, there are three kinds of ways, such as multi-DMD integrated exposure, DMD step exposure and scanning exposure [13, 14]. For the multi-DMD integrated exposure mode [15], on the one hand, the system integration cost is high, which increases the difficulty of data processing. On the other hand, multiple DMD will introduce the interfield splicing error of adjacent DMD and the illumination inconsistency error caused by the parameter difference of different DMD. In step lithography [16], the exposure process is similar to the way of stamping, so its efficiency is not high due to the discontinuous movement of the stage, and perfect stitching between adjacent lithographic edges has always been a problem, which requires a highprecision alignment system to be optimized.

For DMD scanning exposure, the exposure graphs are completed by the dynamic update of DMD pixel and the control of motion stage, so it can realize efficient two-dimensional projection scanning exposure and greatly improve the production capacity. However, based on the dynamic display characteristics of DMD, when DMD displays digital mask graphics, it is actually a process that DMD samples and quantifies the mask according to the pixel size, which will introduce non-integer pixel error, called DMD pixel quantization error. The error will lead to the deviation between the design size and the production size of the mask exposure graphics, resulting in sawtooth defects at the edge of the contour structure of the lithographic images.

In this letter, we proposed an effective fabrication system based on DMD subpixel dynamic scanning lithography, which is also called oblique scanning lithography. Based on this scanning strategy, controlling the position of the exposure and the amount of spot energy accumulated will result in smaller size pixels, which can effectively improve the lithographic images resolution. During the whole system design and construction process, the illumination optical system with excellent uniformity is obtained by ZEMAX optical simulation optimization. Based on pulse width modulation (PWM) technology, the control system of synchronous exposure light source is designed flexibly. Finally, our experiments showed that the developed system can efficiently and accurately fabricate large-area grating images with different line types and line widths.

\section{Design of digital oblique scanning lithography system 2.1. Optical system design}

Fig. 1 shows the schematic diagram of maskless lithography system based on DMD. The system mainly consists of illumination design, projection imaging, DMD graphics control and stage synchronization control. Firstly, the 405nm ultraviolet light-emitting diode (UV-LED) emits ultraviolet light, which is homogenized and collimated through the optical elements. And then, it irradiates on the surface of the DMD Through DMD image control, the reflected light enters the projection imaging lens (CFI Plan Flour $\times 20$, Nikon Co., Japan). Finally, with the control of three-dimensional motion stage (Beijing U-PRECISION TECH CO., LTD, NEWPORT controller), the scanning exposure is realized on the substrate. Here, a Texas Instruments (TI) DMD (model DLP9500) with a 0.95 -inch micromirror array composed of $1920 \times$ 


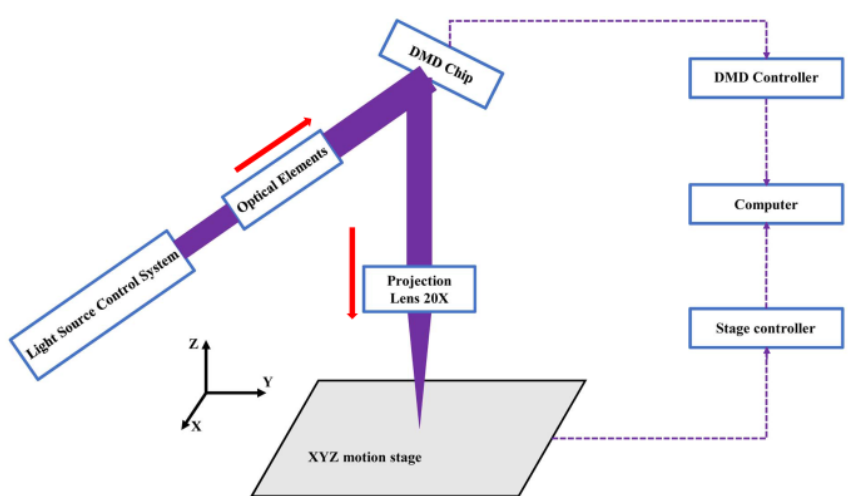

Fig. 1 The schematic diagram of maskless lithography system based on DMD

1080 pixels was adopted, and a single micromirror was $10.8 \mu \mathrm{m} \times$ $10.8 \mu \mathrm{m}$.

\subsection{Light source synchronization control system}

In order to ensure the accuracy and processing efficiency of the exposure pattern, it is necessary to keep the opening and closing of the exposure pattern and the exposure light source synchronized. For this purpose, a light source synchronization control system was designed, as shown in Fig. 2. The control system is a combination of pulse width modulation (PWM) technology and DMD data control technology, which is mainly composed of direct current (DC) stabilized power supply, UV-LED drive board, UV-LED chip, PWM generator and DMD controller. And when the value of PWM generator is below 14 percent, UV-LED will not emit light. So then, light intensity can be adjusted flexibly between 14 and 100 percent under the condition of DMD controller signal output. The system has a large power, which can meet the needs of light energy density in the lithography process. Because of its small shape and size, it is similar to point light source, which is beneficial to the uniform light design of optical system. The calorific value of cold light source is relatively less, which can be used for a long time with the cooling system, especially suitable for continuous production. Compared with traditional high-pressure mercury lamp and laser diode (LD) laser, it has advantages of long service life and low cost.

\begin{tabular}{|l|l|c|}
\hline EN & PWM & UV-LED \\
\hline $0 \mathrm{~V}$ & Any value & No \\
\hline $3.3 \mathrm{~V}$ & $<14 \%$ & No \\
\hline $3.3 \mathrm{~V}$ & $14 \sim 100 \%$ & Yes \\
\hline
\end{tabular}

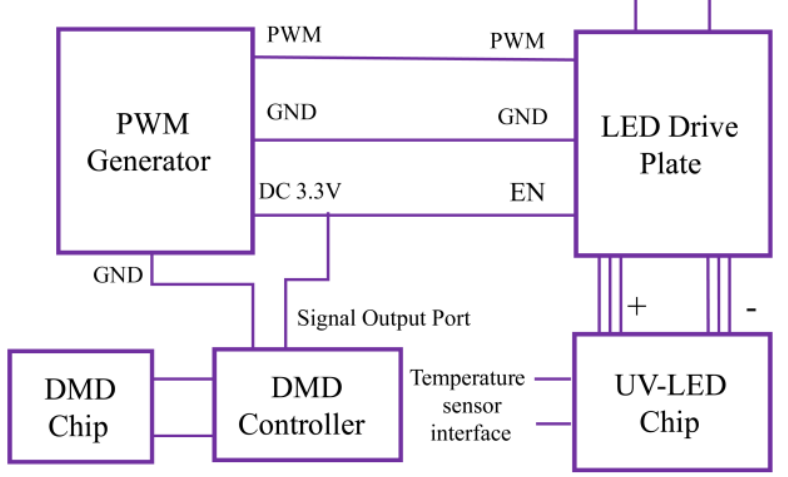

Fig. 2 The schematic diagram of light source control system based on $U V-L E D$

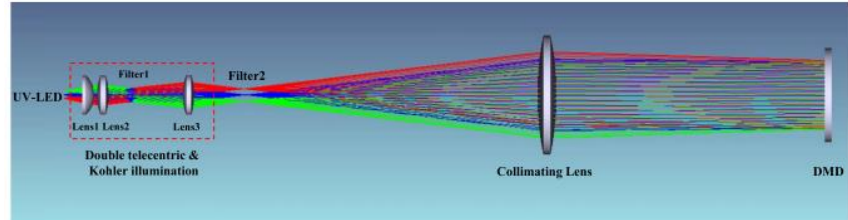

Fig. 3 Light tracing of lighting system by ZEMAX

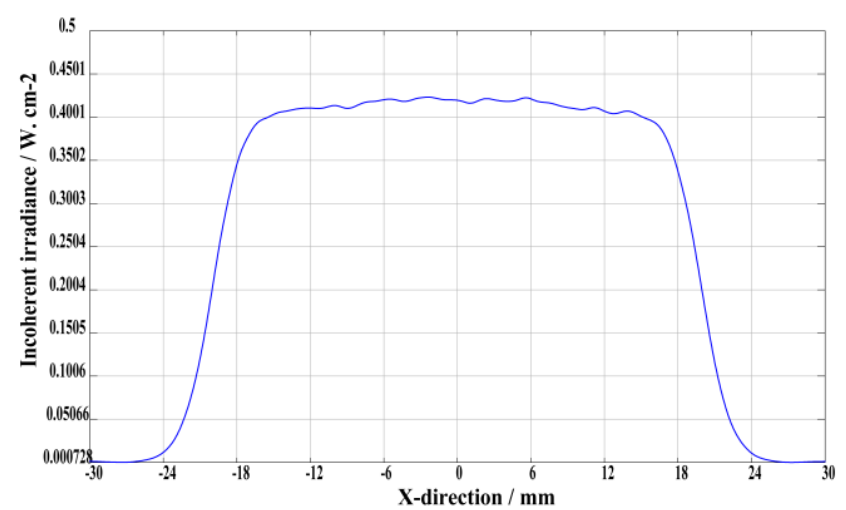

(a)

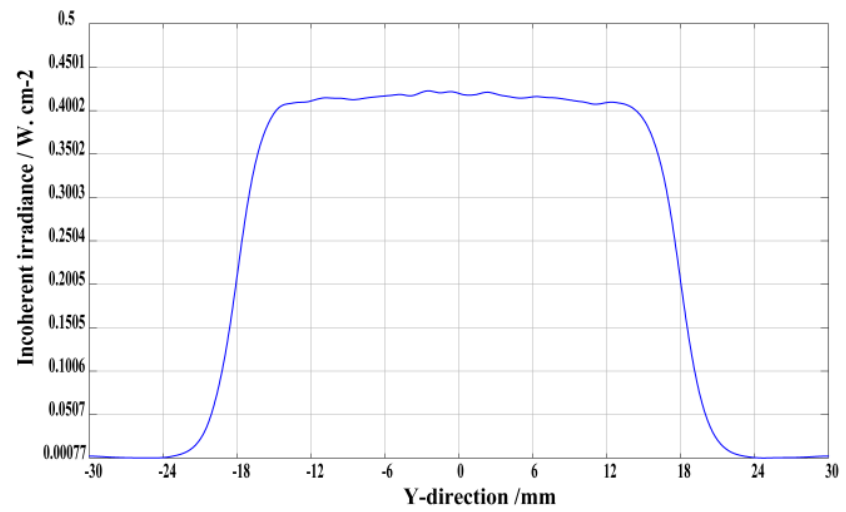

(b)

Fig. 4 Distribution of light intensity on DMD illumination surface (a) X-directional central section

(b) Y-directional central section.

\subsection{Simulation optimization of optical uniformity}

In order to achieve better uniformity of light intensity on the surface of DMD, ZEMAX simulation software for optical path design and non-sequence optimization was adopted. Fig. 3 shows the light tracking diagram of the lighting system. In this design, we directly combined kohler illumination with dual telecentric imaging to construct the illumination system, which was consisted of a series of lenses and a reasonable small hole filter. It aims to make the light homogenized. Then, a collimating lens was combined to ensure the optimum uniformity of the light intensity irradiating on the DMD. In addition, according to the clever design the traditional function of the compound eye lens was replaced, which can effectively avoid the high cost of compound eye lens. What's more, by means of ZEMAX nonsequence optimization, it is easy to obtain the uniform distributions of light intensity in the central section of the DMD illumination surface, as shown in Fig. 4.

It can be seen from Fig. 4(a) and 4(b) that the illumination at the center of $X$ and $Y$ directions is consistent. The following formula is used to describe its uniform consistency, as shown in (1) and (2). 


$$
\begin{aligned}
\operatorname{Uniformity}(X) & =\frac{2 I_{\min }}{I_{\max }+I_{\min }}=\frac{2 \times 0.4059}{0.4228+0.4059} \times 100 \% \\
& =97.96 \%
\end{aligned}
$$

$$
\begin{aligned}
\operatorname{Uniformity}(Y) & =\frac{2 I_{\min }}{I_{\max }+I_{\min }}=\frac{2 \times 0.4047}{0.4240+0.4047} \times 100 \% \\
& =97.67 \%
\end{aligned}
$$

where $I_{\min }$ and $I_{\max }$ are the minimum and maximum light intensity in the illumination central section.

\subsection{Oblique scanning strategy based on DMD}

Fig. 5 is the schematic diagram of the proposed DMD oblique scanning to achieve the sub-pixel grid precision. The DMD chip is rotated to form a $\theta$-degree angle with the scanning direction, and then smaller size pixels are formed by using the exposure position and the amount of spot integral energy, so as to achieve the sub-pixel exposure accuracy. Assuming that the micromirror spacing of DMD is $P W 1$, it can be known that the grid precision before DMD is also $P W 1$, while the grid precision $P W 2$ after DMD is obviously smaller than that before DMD is tilted. In addition, the relationship between the tilt factor $(\mathrm{N})$, tilt angle $(\theta)$ and mesh precision $(P W 2)$ in the Fig. 5 is obvious, and there exists the following geometric relationship in (3).

$$
P W 2=d \times \sin \theta=\frac{d}{\sqrt{N^{2}+1}}
$$

That is, the larger the tilt factor is, the smaller the corresponding tilt angle is, and the higher the mesh precision will be.

Further analysis based on the principle of DMD dynamic scanning lithography shows that the number of sub-pixel superimposed spots in the scanning direction and step direction of the motion stage is determined by the scanning speed of the moving platform and the tilt angle of DMD. The number of spot overlaps $O_{\text {scan }}$ in the scanning direction can be expressed by the following (4).

$$
O_{\text {scan }}=\left(1-\frac{V}{F \cdot d}\right) \times 100
$$

where $F$ is the DMD frame frequency, $V$ is the scanning speed of the motion stage, $d$ is the spot diameter.

The number of overlap spots in the step direction, combined with

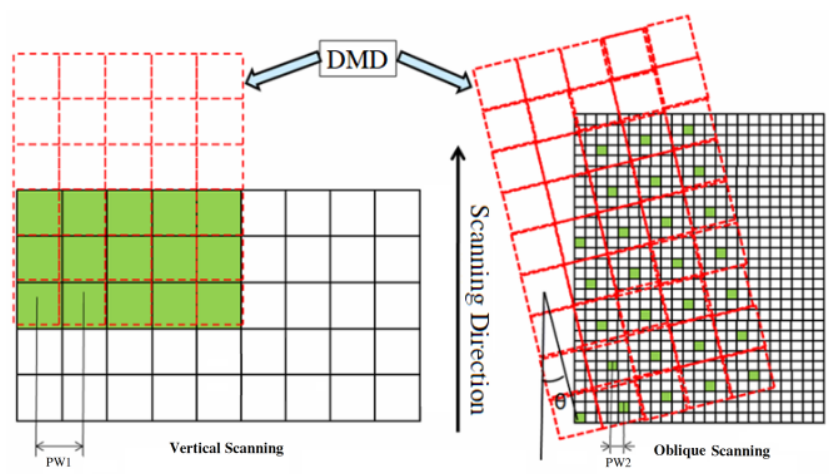

Fig. 5 Schematic diagram of the realization of subpixel lithography by oblique scanning strategy
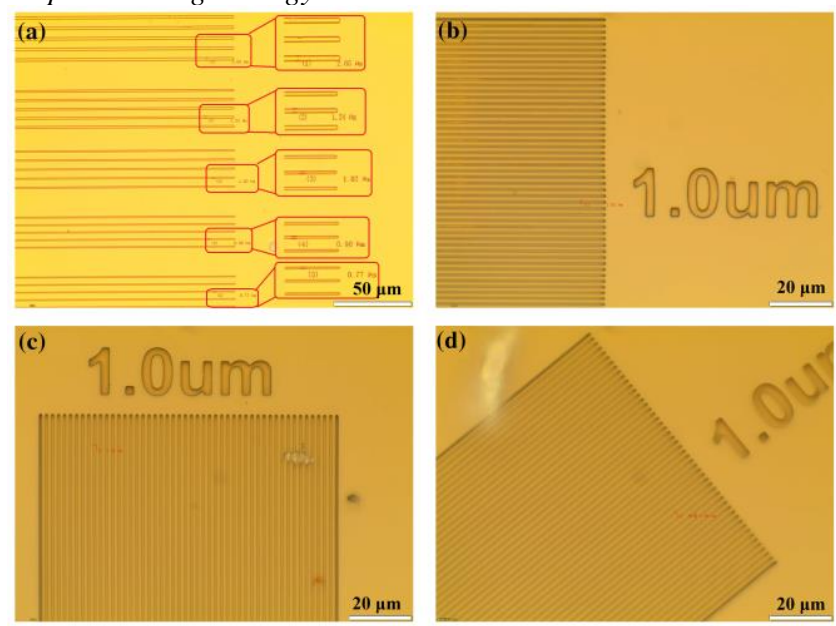

Fig. 6 Experimental verification of lithography exposure with different lines widths and lines types

(a) Horizontal lines of $0.8 \mu \mathrm{m} \sim 2 \mu \mathrm{m}$.

(b) Horizontal lines of $1 \mu \mathrm{m}$.

(c) Vertical lines of $1 \mu \mathrm{m}$

(d) $45^{\circ}$ lines of $1 \mu \mathrm{m}$

$P W 2=d \times \sin \theta$, can be deduced as follows in (5).

$$
O_{\text {step }}=\left(1-\frac{P W 2}{d}\right) \times 100
$$

In the experimental study, we set the tilt factor $N$ to 8 , and then obtained the tilt angle $\theta=7.125^{\circ}$ by trigonometric calculation. According to $P W 2$ expression, the sub-pixel resolution can be calculated. when $P W 2=1.340 \mu \mathrm{m}$. Obviously, the pixel resolution is significantly higher than before the tilt.

\section{Experimental verification}

According to the design of digital oblique scanning lithography system above, the fabrication quality can be easily guaranteed. Moreover, we verified the rationality and effectiveness of the designed system through experiments, as shown in Fig. 6. Fig. 6(a) showed the horizonal lines with $0.8 \mu \mathrm{m} \sim 2.0 \mu \mathrm{m}$ line widths. It can be seen that the measured results were very close to the theoretical line widths. And then, to further verify the processing effect of images in different directions, three kinds of directions lines with $1 \mu \mathrm{m}$ line widths were fabricated, respectively. Fig. (b), (c) and (d) are the horizonal lines, vertical lines, 45-degree slope lines, respectively. Observing the results of exposure, it can be found that the overall line widths of the three directions is the same and the uniformity is better. Compare with traditional maskless scanning lithography, there is no sawtooth structures on the edge of the exposure images, and the edge of grating structures is smooth.

\section{Conclusions}

In summary, we have successfully developed an effective maskless lithography system. Combined with the optimization method of ZEMAX optical design, the best uniformity of optical lighting is obtained. The UV-LED light source synchronization control system was developed, which can effectively reduce the exposure pattern error and improve the exposure efficiency. Moreover, based on digital oblique scanning strategy the lithography resolution can be effectively 
improved and eliminate the effect of pixel quantization error. Finally, the effectiveness of the proposed lithography system is verified by experiments, which is expected to be used for welding and packaging of large-area PCB and applied to three-dimensional micro-nano structure devices.

\section{Acknowledgments}

This work is supported by funding from the Key project of natural science research of Anhui Province (KJ2019A0156); Chinese National Natural Science Foundation (51475442); Natural Science Foundation of Anhui Province of China (1808085ME117, 1708085ME129); Scientific Research Foundation for the Introduction of Talent of Anhui Polytechnic University (2018YQQ027, 2017YQQ008); Domestic visiting and training program for outstanding young backbone talents from universities and colleges (gxgnfx2019013); Science and Technology Program of Wuhu, China (2018yf55); Young and middle-aged top talent project of Anhui Polytechnic University; The youth top-notch talent of Anhui Polytechnic University.

\section{References}

[1] Y. Liao, J. Song, E. Li, Y. Luo, Y. Shen, D. Chen, Y. Cheng, Z. Xu, K. Sugioka and K. Midorikawa: Lab Chip, 12, (2012) 746.

[2] Y. Chen: Microelectron. Eng, 135, (2015) 57.

[3] M. Gschrey, A. Thoma, P. Schnauber, M. Seifried, R. Schmidt, B. Wohlfeil, L. Krüger, J.-H. Schulze, T. Heindel and S. Burger: Nat. Commun, 6, (2015) 1.

[4] D.H. Dinh, H.L. Chien and Y.C. Lee: Opt. Laser. Technol, 113, (2019) 407.

[5] J.B. Kim and K.H. Jeong: Opt. Express, 25, (2017) 16854.

[6] S. Huang, M. Li, L. Shen, J. Qiu and Y. Zhou: Micro. Nano. Lett, 12 , (2017) 49.

[7] Q. Deng, Y. Yang, H. Gao, Y. Zhou, Y. He and S. Hu: Micromachines-Basel, 8, (2017) 314.

[8] K. Kim, S. Han, J. Yoon, S. Kwon, H.K. Park and W. Park: Appl. Phys. Lett, 109, (2016) 234101.

[9] J. Lee, H. Lee and J. Yang: J. Mech. Sci. Technol, 32, (2018) 2209.

[10] S. TOMOEDA, Y. GOTO, D. KUMAKI, S. TOKITO, T. HATAKEYAMA and H. HIRATA: Nihon. Gazo. Gakk, 56, (2017) 611.

[11] A. Bertsch, H. Lorenz and P. Renaud: Sensor. Actuat. A-Phys, $73,(1999) 14$.

[12] W. Iwasaki, T. Takeshita, Y. Peng, H. Ogino, H. Shibata, Y. Kudo, R. Maeda and R. Sawada: Jpn. J. Appl. Phys, 51, (2012) 06FB05.

[13] R. Chen, H. Liu, H. Zhang, W. Zhang, J. Xu, W. Xu and J. Li: Opt. Express, 25, (2017) 21958.
[14] H. Ryoo, D.W. Kang and J.W. Hahn: Microelectron. Eng, 88, (2011) 3145 .

[15] J.H.R. Chang, B.V. Kumar and A.C. Sankaranarayanan: Opt. Express, 24, (2016) 27937.

[16] H. Wu, W. Hu, H.C. Hu, X.W. Lin, G. Zhu, J.W. Choi, V. Chigrinov and Y.Q. Lu: Opt. Express, 20, (2012) 16684.

(Received: January 12, 2020, Accepted: March 23, 2020) 